QUARTERLY OF APPLIED MATHEMATICS

VOLUME LXV, NUMBER 3

SEPTEMBER 2007, PAGES 549-569

S $0033-569 X(07) 01054-0$

Article electronically published on April 19, 2007

\title{
ON PROPERTIES OF SOME INTEGRALS RELATED TO POTENTIALS FOR STOKES EQUATIONS
}

\author{
BY \\ P. A. KRUTITSKII
}

Department of Mathematics, Faculty of Physics, Moscow State University, Moscow 119899, Russia

\begin{abstract}
The integrals arising from potentials for two-dimensional Stokes equations are explored in the case when the potentials are defined on the smooth open arc of an arbitrary shape, while the densities in the potentials belong to weighted Hölder space and may have power singularities. The properties of smoothness of these integrals and their derivatives are studied. The singularities of the derivatives of the integrals at the ends of the arcs are examined. The integrals studied in the paper being coupled with harmonic logarithmic potential yield single layer potentials for velocities in Stokes equations. Single layer potential for pressure in Stokes equations is investigated also.
\end{abstract}

1. Introduction. Potential theory for $2 \mathrm{D}$ Stokes equations has been constructed in 2 in the case of smooth closed curves and continuous densities. This potential theory has been applied in 2] to the analysis of solvability of boundary value problems for Stokes equations in the interior and exterior of simply connected domains bounded by a smooth closed curve. Results obtained in [2] have been extended in [3, 8, 9, to the case of multiply connected domains bounded by smooth closed curves. Boundary value problems in [3, 8, 9] are reduced to uniquely solvable integral equations. The present paper is devoted to the analysis of some functions presented in the form of integrals related to Stokes potentials specified on a nonclosed arc in the case when the densities in the potentials are taken in weighted Hölder space and may have power singularities. Smoothness properties of these functions and the smoothness of their derivatives are studied. Singularities of the derivatives of these functions at the ends of the arc are investigated. It should be noted that the integral functions studied in the paper being coupled with a logarithmic harmonic potential yield single layer potentials for Stokes velocities. The basic difficulties in the analysis of these integral functions follow from the fact that they are not harmonic unlike logarithmic potential. In the last section of the paper the single layer Stokes potential for pressure is analyzed.

Received November 15, 2006.

2000 Mathematics Subject Classification. Primary 31A10, 35Q30.

The research was supported by the RFBR grants 05-01-00050, 07-01-00029 and the Bernoulli Center in Lausanne (Switzerland).

(C)2007 Brown University 
2. Formulation of the problem. By an open arc we mean a simple smooth nonclosed arc of finite length without self-intersections [1]. In Cartesian coordinates $x=$ $\left(x_{1}, x_{2}\right) \in \mathbb{R}^{2}$ in a plane we consider a simple smooth open $\operatorname{arc} \Gamma$ parametrized by the arc length $s: \Gamma=\left\{x: x=x(s)=\left(x_{1}(s), x_{2}(s)\right), s \in[a, b]\right\}$. Therefore points $x \in \Gamma$ and values of the parameter $s$ are in one-to-one correspondence. We denote the tangent vector to $\Gamma$ at the point $x(s)$ by $\tau_{s}=(\cos \alpha(s), \sin \alpha(s))$, where $\cos \alpha(s)=x_{1}^{\prime}(s)$, $\sin \alpha(s)=x_{2}^{\prime}(s)$. Let $\mathbf{n}_{s}=(\sin \alpha(s),-\cos \alpha(s))$ be a normal vector to $\Gamma$ at $x(s)$. The direction of $\mathbf{n}_{s}$ is chosen such that it will coincide with the direction of $\tau_{s}$ if $\mathbf{n}_{s}$ is rotated counterclockwise through an angle of $\pi / 2$. The segment $[a, b]$ will be denoted by $\Gamma$ also.

Let the real-valued function $\mu(s)$ of class $\mathbb{C}_{q}^{\omega}[a, b], \omega \in(0,1], q \in[0,1)$ be specified on $\Gamma$. We say that $\mu(s) \in \mathbb{C}_{q}^{\omega}[a, b]$ if $\mu_{0}(s) \in \mathbb{C}^{0, \omega}[a, b]$, where $\mu_{0}(s)=\mu(s)|s-a|^{q}|s-b|^{q}$, and $\mathbb{C}^{0, \omega}[a, b]$ is a Hölder class with the exponent $\omega$.

We consider $\Gamma$ as a cut in a plane. The side of the cut $\Gamma$ that is on the left when the parameter $s$ increases will be denoted by $\Gamma^{+}$and the opposite side we denote by $\Gamma^{-}$. In a similar manner, by the superscripts "+" and "-" we will denote the limit values of functions on $\Gamma^{+}$and $\Gamma^{-}$respectively.

The aim of the present paper is to study properties of some integrals, which appear in the analysis of the vector single layer potential for the Stokes equations [2, 3]. Consider the integrals

$$
\begin{aligned}
& \mathcal{C}[\mu](x)=\frac{1}{2 \pi} \int_{\Gamma} \mu(\sigma) \cos (2 \psi(x, y(\sigma))) d \sigma, \\
& \mathcal{S}[\mu](x)=\frac{1}{2 \pi} \int_{\Gamma} \mu(\sigma) \sin (2 \psi(x, y(\sigma))) d \sigma,
\end{aligned}
$$

where $\cos (2 \psi)=\cos ^{2}(\psi)-\sin ^{2}(\psi), \quad \sin (2 \psi)=2 \cos (\psi) \sin (\psi)$,

$$
\begin{gathered}
\cos \psi(x, y(\sigma))=\frac{x_{1}-y_{1}(\sigma)}{|x-y(\sigma)|}, \quad \sin \psi(x, y(\sigma))=\frac{x_{2}-y_{2}(\sigma)}{|x-y(\sigma)|}, \\
y(\sigma)=\left(y_{1}(\sigma), y_{2}(\sigma)\right) \in \Gamma, \quad|x-y(\sigma)|=\sqrt{\left(x_{1}-y_{1}(\sigma)\right)^{2}+\left(x_{2}-y_{2}(\sigma)\right)^{2}} .
\end{gathered}
$$

Let $\quad z=x_{1}+i x_{2}, \quad t=t(\sigma)=\left(y_{1}(\sigma)+i y_{2}(\sigma)\right) \in \Gamma$. Then $\quad d t=t^{\prime}(\sigma) d \sigma=e^{i \alpha(\sigma)} d \sigma$, $z-t(\sigma)=|x-y(\sigma)| e^{i \psi(x, y(\sigma))}, \quad \bar{z}-\bar{t}(\sigma)=|x-y(\sigma)| e^{-i \psi(x, y(\sigma))}$. Complex conjugate values are denoted by the overline. We introduce the function

$$
\begin{gathered}
\mathcal{E}[\mu](x)=\mathcal{C}[\mu](x)-i \mathcal{S}[\mu](x) \\
=\frac{1}{2 \pi} \int_{\Gamma} \mu(\sigma) e^{-2 i \psi(x, y(\sigma))} d \sigma=\frac{1}{2 \pi} \int_{\Gamma} \mu(\sigma) \frac{\bar{z}-\bar{t}(\sigma)}{z-t(\sigma)} d \sigma \\
=\frac{1}{2 \pi} \int_{\Gamma} \mu(\sigma) e^{-i \alpha(\sigma)} \frac{\bar{z}-\bar{t}(\sigma)}{z-t(\sigma)} d t=\frac{1}{2 \pi} \int_{\Gamma} \hat{\mu}(t) \frac{\bar{t}-\bar{z}}{t-z} d t,
\end{gathered}
$$

where $\hat{\mu}(t)=\mu(\sigma) e^{-i \alpha(\sigma)}$.

We say that the complex-valued function $\hat{\mu}(t)$ belongs to the class $\mathbb{C}_{q}^{\omega}(\Gamma)$ with $\omega \in$ $(0,1], q \in[0,1)$ if $\hat{\mu}_{0}(t) \in \mathbb{C}^{0, \omega}(\Gamma)$, where $\hat{\mu}_{0}(t)=\hat{\mu}(t)|t-t(a)|^{q}|t-t(b)|^{q}$, and $\mathbb{C}^{0, \omega}(\Gamma)$ is a Hölder class with the exponent $\omega$.

Now we study some properties of functions specified on a smooth open arc. 
Lemma 1. Let $\Gamma$ be a smooth open arc. Let $g(\sigma) \in \mathbb{C}^{0, \omega}[a, b], \omega \in(0,1]$, and $\hat{g}(t)=g(\sigma)$, $t=t(\sigma)=\left(y_{1}(\sigma)+i y_{2}(\sigma)\right) \in \Gamma$. Then $\hat{g}(t) \in \mathbb{C}^{0, \omega}(\Gamma)$.

Proof. Let $t_{1}, t_{2} \in \Gamma ; t_{k}=t\left(\sigma_{k}\right)=y_{1}\left(\sigma_{k}\right)+i y_{2}\left(\sigma_{k}\right), k=1,2$. Then

$$
\left|\hat{g}\left(t_{2}\right)-\hat{g}\left(t_{1}\right)\right|=\left|g\left(\sigma_{2}\right)-g\left(\sigma_{1}\right)\right| \leq c\left|\sigma_{2}-\sigma_{1}\right|^{\omega} .
$$

It follows from Lemma 1 in 4 that the function $\left|\frac{\sigma_{2}-\sigma_{1}}{t_{2}-t_{1}}\right|=\left|\frac{\sigma_{2}-\sigma_{1}}{t\left(\sigma_{2}\right)-t\left(\sigma_{1}\right)}\right|$ is continuous on $[a, b]$ in both variables $\sigma_{1}$ and $\sigma_{2}$, and so, it is uniformly bounded, i.e.,

$$
\left|\frac{\sigma_{2}-\sigma_{1}}{t_{2}-t_{1}}\right| \leq \text { const }
$$

for any $\sigma_{1}, \sigma_{2} \in[a, b]$. Therefore

$$
\left|\hat{g}\left(t_{2}\right)-\hat{g}\left(t_{1}\right)\right| \leq c\left|\frac{\sigma_{2}-\sigma_{1}}{t_{2}-t_{1}}\right|^{\omega}\left|t_{2}-t_{1}\right|^{\omega} \leq c_{1}\left|t_{2}-t_{1}\right|^{\omega},
$$

where $c_{1}$ is a constant. Hence, $\hat{g}(t)$ satisfies the Hölder inequality on $\Gamma$ in the $t$ variable with the exponent $\omega$. The proof is completed.

Using Lemma 1 we can prove

Lemma 2. Let $\Gamma$ be an open arc of class $\mathbb{C}^{1, \lambda}, \lambda \in(0,1]$, and

$$
t=t(\sigma)=\left(y_{1}(\sigma)+i y_{2}(\sigma)\right) \in \Gamma .
$$

1) If $f(\sigma) \in \mathbb{C}^{0, \omega}[a, b], \omega \in(0,1]$ and if $\hat{f}(t)=f(\sigma) e^{-i \alpha(\sigma)}$, then $\hat{f}(t) \in \mathbb{C}^{0, \beta}(\Gamma)$, $\beta=\min \{\omega, \lambda\}$.

2) If $\mu(\sigma) \in \mathbb{C}_{q}^{\omega}[a, b], \quad \omega \in(0,1], \quad q \in[0,1)$, and if $\hat{\mu}(t)=\mu(\sigma) e^{-i \alpha(\sigma)}$, then $\hat{\mu}(t) \in \mathbb{C}_{q}^{\beta}(\Gamma), \beta=\min \{\omega, \lambda\}$.

Proof. 1) Clearly, $e^{-i \alpha(\sigma)} \in \mathbb{C}^{0, \lambda}[a, b]$. According to the properties of Hölder functions [1], $f(\sigma) e^{-i \alpha(\sigma)} \in \mathbb{C}^{0, \beta}[a, b]$. Now the statement of point 1) in this lemma follows from Lemma 1. Let us prove point 2). Set $\mu_{0}(\sigma)=\mu(\sigma)|\sigma-a|^{q}|\sigma-b|^{q}$ and $t=t(\sigma)$. Then

$$
\begin{gathered}
\hat{\mu}_{0}(t)=\hat{\mu}(t)|t-t(a)|^{q}|t-t(b)|^{q} \\
=\mu(\sigma) e^{-i \alpha(\sigma)}\left(\frac{|t(\sigma)-t(a)|}{|\sigma-a|} \frac{|t(\sigma)-t(b)|}{|\sigma-b|}\right)^{q}|\sigma-a|^{q}|\sigma-b|^{q} \\
=\mu_{0}(\sigma) e^{-i \alpha(\sigma)}\left|\frac{t(\sigma)-t(a)}{\sigma-a}\right|^{q}\left|\frac{t(\sigma)-t(b)}{\sigma-b}\right|^{q} .
\end{gathered}
$$

It follows from [4, Lemma 1] that the functions $\left|\frac{t(\sigma)-t(a)}{\sigma-a}\right|$ and $\left|\frac{t(\sigma)-t(b)}{\sigma-b}\right|$ belong to $\mathbb{C}^{0, \lambda}[a, b]$ in $\sigma$ and they are not equal to zero for any $\sigma \in[a, b]$. On the basis of the properties of Hölder functions presented in [1] we observe that the functions $\left|\frac{t(\sigma)-t(a)}{\sigma-a}\right|^{q}$ and $\left|\frac{t(\sigma)-t(b)}{\sigma-b}\right|^{q}$ belong to $\mathbb{C}^{0, \lambda}[a, b]$ in $\sigma$, since the function $r^{q}$ is of class $\mathbb{C}^{1}$ on any segment that does not contain the point $r=0$. Note that $\mu_{0}(\sigma) \in \mathbb{C}^{0, \omega}[a, b]$, since 
$\mu(\sigma) \in \mathbb{C}_{q}^{\omega}[a, b]$. Consequently, using the properties of Hölder functions [1, we obtain

$$
\mu_{1}(\sigma)=\mu_{0}(\sigma)\left|\frac{t(\sigma)-t(a)}{\sigma-a}\right|^{q}\left|\frac{t(\sigma)-t(b)}{\sigma-b}\right|^{q} \in \mathbb{C}^{0, \beta}[a, b] .
$$

Hence $\hat{\mu}_{0}(t)=\mu_{1}(\sigma) e^{-i \alpha(\sigma)}$ from (1). Using point 1) of this lemma, where $f(\sigma) \equiv \mu_{1}(\sigma)$ and $\hat{f}(t)=\hat{\mu}_{0}(t)$, we obtain that $\hat{\mu}_{0}(t) \in \mathbb{C}^{0, \beta}(\Gamma)$. It follows from $(1)$ that $\hat{\mu}(t)$ belongs to the class $\mathbb{C}_{q}^{\beta}(\Gamma)$ according to the definition of this class. The proof is completed.

We say that the function of several variables satisfies the Hölder inequality if it satisfies the Hölder inequality in each variable uniformly with respect to the other variables [1, sect. 3.2].

From Lemma 1 we obtain

Lemma 3. Let $\Gamma$ be a smooth open arc. Let $g(s, \sigma) \in \mathbb{C}^{0, \omega}([a, b] \times[a, b]), \omega \in(0,1]$ and $\hat{g}\left(t_{0}, t\right)=g(s, \sigma), \quad t_{0}=t_{0}(s)=\left(x_{1}(s)+i x_{2}(s)\right) \in \Gamma, \quad t=t(\sigma)=\left(y_{1}(\sigma)+i y_{2}(\sigma)\right) \in \Gamma$. Then $\hat{g}\left(t_{0}, t\right) \in \mathbb{C}^{0, \omega}(\Gamma \times \Gamma)$.

3. Properties of integrals. The aim of this section is to study properties of the integrals $\mathcal{C}[\mu](x)$ and $\mathcal{S}[\mu](x)$.

Lemma 4. Let $\Gamma$ be an open arc of class $\mathbb{C}^{1, \lambda}, \lambda \in(0,1]$. Then

1) the functions $\cos (2 \psi(x(s), y(\sigma))), \sin (2 \psi(x(s), y(\sigma)))$ belong to the class $\mathbb{C}^{0, \lambda}([a, b] \times[a, b])$ in $s$ and $\sigma$;

2) the function $\hat{g}\left(t_{0}, t\right)=\frac{\bar{t}-\bar{t}_{0}}{t-t_{0}}$ belongs to the class $\mathbb{C}^{0, \lambda}(\Gamma \times \Gamma)$ in $t, t_{0}$.

Proof. Note that

$$
\begin{gathered}
\cos (2 \psi(x(s), y(\sigma)))=1-2 \sin ^{2} \psi(x(s), y(\sigma)), \\
\sin (2 \psi(x(s), y(\sigma)))=2 \cos \psi(x(s), y(\sigma)) \sin \psi(x(s), y(\sigma)) .
\end{gathered}
$$

Hence, to prove point 1) it is sufficient to prove that the functions $\sin ^{2} \psi(x(s), y(\sigma))$ and $[\cos \psi(x(s), y(\sigma)) \sin \psi(x(s), y(\sigma))]$ belong to the class $\mathbb{C}^{0, \lambda}([a, b] \times[a, b])$ in $s, \sigma$. We can prove this fact using the formulae

$$
\cos \psi(x(s), y(\sigma))=\frac{x_{1}(s)-y_{1}(\sigma)}{|x(s)-y(\sigma)|}, \quad \sin \psi(x(s), y(\sigma))=\frac{x_{2}(s)-y_{2}(\sigma)}{|x(s)-y(\sigma)|},
$$

where

$$
y(\sigma)=\left(y_{1}(\sigma), y_{2}(\sigma)\right) \in \Gamma,|x-y(\sigma)|=\sqrt{\left(x_{1}-y_{1}(\sigma)\right)^{2}+\left(x_{2}-y_{2}(\sigma)\right)^{2}},
$$

and using the technique developed in Lemma 1 in 4 .

Point 2) of the lemma follows from point 1) and from Lemma 3 if we take into account that

$$
\begin{gathered}
\hat{g}\left(t_{0}, t\right)=\frac{\bar{t}-\bar{t}_{0}}{t-t_{0}}=e^{-2 i \psi(x(s), y(\sigma))} \\
=\cos (2 \psi(x(s), y(\sigma)))-i \sin (2 \psi(x(s), y(\sigma)))=g(s, \sigma),
\end{gathered}
$$


where $t_{0}=t_{0}(s)=\left(x_{1}(s)+i x_{2}(s)\right) \in \Gamma, t=t(\sigma)=\left(y_{1}(\sigma)+i y_{2}(\sigma)\right) \in \Gamma$. Indeed, since $g(s, \sigma)$ belongs to $\mathbb{C}^{0, \lambda}([a, b] \times[a, b])$ according to point 1$)$, it follows from Lemma 3 that $\hat{g}\left(t_{0}, t\right) \in \mathbb{C}^{0, \lambda}(\Gamma \times \Gamma)$.

Lemma 5. Let $\Gamma$ be an open arc of class $\mathbb{C}^{1, \lambda}, \lambda \in(0,1]$. Let $\mu(s) \in \mathbb{C}_{q}^{\omega}[a, b], \omega \in(0,1]$, $q \in[0,1)$. Then the functions $\mathcal{C}[\mu](x(s)), \mathcal{S}[\mu](x(s))$ belong to the class $\mathbb{C}^{0, \lambda}[a, b]$ in $s$.

The proof can be given by direct verification with using point 1) of Lemma 4 .

Lemma 6 . Let $\Gamma$ be an open arc of class $\mathbb{C}^{1, \lambda}, \lambda \in(0,1]$ and let an arc $\Gamma^{\prime}$ be an arbitrary part of $\Gamma$ such that the ends of $\Gamma^{\prime}$ are interior points of $\Gamma$. Let $\hat{\mu}(t) \in \mathbb{C}_{q}^{\omega}(\Gamma), \omega \in(0,1]$, $q \in[0,1)$. Then the singular integral

$$
I[\hat{\mu}]\left(t_{0}\right)=\frac{1}{2 \pi i} \int_{\Gamma} \frac{\hat{\mu}(t)}{t-t_{0}} \frac{\bar{t}-\bar{t}_{0}}{t-t_{0}} d t
$$

belongs to the class $\mathbb{C}^{0, \beta_{0}}\left(\Gamma^{\prime}\right)$ in the $t_{0}$ variable for any $\beta_{0} \in(0, \beta)$, where $\beta=\min \{\omega, \lambda\}$.

Proof. Let an open arc $\Gamma^{\prime \prime}$ be a part of $\Gamma$ such that the endponts of $\Gamma^{\prime \prime}$ are interior points of $\Gamma$ and all points of $\Gamma^{\prime}$ (including endpoints) are interior points of $\Gamma^{\prime \prime}$. Evidently, $\hat{\mu}(t) \in \mathbb{C}^{0, \omega}\left(\Gamma^{\prime \prime}\right)$. According to point 2$)$ of Lemma 4 , the function $\hat{g}\left(t_{0}, t\right)=\frac{\bar{t}-\bar{t}_{0}}{t-t_{0}}$ belongs to the class $\mathbb{C}^{0, \lambda}(\Gamma \times \Gamma)$ in $t, t_{0}$. Then the function $\hat{\mu}(t) \hat{g}\left(t_{0}, t\right)$ belongs to $\mathbb{C}^{0, \beta}\left(\Gamma^{\prime \prime} \times \Gamma^{\prime \prime}\right)$ in $t_{0}, t$. Now the statement of the lemma follows from the properties of singular integrals, the density of which depends on a parameter [1, sect. 18.4].

TheOREm 1. If $\Gamma$ is an open arc of class $\mathbb{C}^{1, \lambda}, \lambda \in(0,1]$, and $\mu(\sigma) \in \mathbb{C}_{q}^{\omega}[a, b]$, with $\omega \in(0,1], q \in[0,1)$, then $\mathcal{C}[\mu](x) \in \mathbb{C}^{0}\left(\mathbb{R}^{2}\right)$ and $\mathcal{S}[\mu](x) \in \mathbb{C}^{0}\left(\mathbb{R}^{2}\right)$.

Proof. If $\hat{\mu}(t)=\mu(\sigma) e^{-i \alpha(\sigma)}$, then $\hat{\mu}(t) \in \mathbb{C}_{q}^{\beta}(\Gamma)$ for $\beta=\min \{\omega, \lambda\}$, according to point 2) of Lemma 2. It follows from the properties of integrals of Cauchy type [1] that the function

$$
\mathcal{E}[\mu](x)=\frac{1}{2 \pi}\left\{\int_{\Gamma} \hat{\mu}(t) \frac{\bar{t}}{t-z} d t-\bar{z} \int_{\Gamma} \hat{\mu}(t) \frac{1}{t-z} d t\right\}, \quad z=x_{1}+i x_{2},
$$

is continuously extensible from $\mathbb{R}^{2} \backslash \Gamma$ on $\Gamma^{+}$and on $\Gamma^{-}$to the interior points of $\Gamma$. The limit values of a function $\mathcal{E}[\mu](x)$ on $\Gamma^{+}$and on $\Gamma^{-}$in the point $x^{0} \in \Gamma$ are denoted by $\mathcal{E}^{+}[\mu]\left(x^{0}\right)$ and $\mathcal{E}^{-}[\mu]\left(x^{0}\right)$ respectively. According to [1, Section 15], we obtain that $\mathcal{E}^{+}[\mu]\left(x^{0}\right) \in \mathbb{C}^{0}(\Gamma \backslash\{x(a) \cup x(b)\})$ and $\mathcal{E}^{-}[\mu]\left(x^{0}\right) \in \mathbb{C}^{0}(\Gamma \backslash\{x(a) \cup x(b)\})$. Now we use the Plemelj formulae [1] and derive limiting values $\mathcal{E}^{+}[\mu]\left(x^{0}\right)$ and $\mathcal{E}^{-}[\mu]\left(x^{0}\right)$ on $\Gamma^{+}$and $\Gamma^{-}$ respectively in the point $x^{0}=\left(x_{1}^{0}, x_{2}^{0}\right)$, which is an interior point of $\Gamma$. Then we obtain

$$
\begin{aligned}
\mathcal{E}^{ \pm}[\mu]\left(x^{0}\right) & =i\left\{\left[\frac{1}{2 \pi i} \int_{\Gamma} \hat{\mu}(t) \frac{\bar{t} d t}{t-z}\right]^{ \pm}-\left[\frac{\bar{z}}{2 \pi i} \int_{\Gamma} \hat{\mu}(t) \frac{d t}{t-z}\right]^{ \pm}\right\} \\
=i\left\{ \pm \frac{1}{2} \hat{\mu}\left(t_{0}\right) \bar{t}_{0}\right. & \left.+\frac{1}{2 \pi i} \int_{\Gamma} \hat{\mu}(t) \frac{\bar{t} d t}{t-t_{0}}-\left[ \pm \frac{1}{2} \hat{\mu}\left(t_{0}\right) \bar{t}_{0}+\frac{\bar{t}_{0}}{2 \pi i} \int_{\Gamma} \hat{\mu}(t) \frac{d t}{t-t_{0}}\right]\right\} \\
& =\frac{1}{2 \pi} \int_{\Gamma} \hat{\mu}(t) \frac{\bar{t}-\bar{t}_{0}}{t-t_{0}} d t, \quad t_{0}=x_{1}^{0}+i x_{2}^{0} .
\end{aligned}
$$


Consequently, $\mathcal{E}^{+}[\mu]\left(x^{0}\right)=\mathcal{E}^{-}[\mu]\left(x^{0}\right)=\mathcal{E}[\mu]\left(x^{0}\right)$ in the interior points of $\Gamma$, where $\mathcal{E}[\mu]\left(x^{0}\right)$ is the direct value of $\mathcal{E}[\mu](x)$ on $\Gamma$. Therefore, $\mathcal{E}[\mu](x) \in \mathbb{C}^{0}\left(\mathbb{R}^{2} \backslash\{x(a) \cup x(b)\}\right)$. Using the Lebesgue theorem on passing to the limit under the Lebesgue integral [5, sect. 1.4], one can verify that $\mathcal{E}[\mu](x)$ is continuously extensible at the ends of $\Gamma$ if $x$ tends to the end of $\Gamma$ from $\mathbb{R}^{2} \backslash \Gamma$. It follows from Lemmas 5 and 1 that the functions $\mathcal{C}[\mu](x)$ and $\mathcal{S}[\mu](x)$ belong to $\mathbb{C}^{0, \lambda}(\Gamma)$ in $x$. Therefore $\mathcal{E}[\mu](x) \in \mathbb{C}^{0}\left(\mathbb{R}^{2}\right)$ and we obtain the statement of the theorem.

4. Properties of the derivatives of the integrals. Let $z=x_{1}+i x_{2}, \quad t=$ $t(\sigma)=\left(y_{1}(\sigma)+i y_{2}(\sigma)\right) \in \Gamma$. Then $z-t(\sigma)=|x-y(\sigma)| e^{i \psi(x, y(\sigma))}$ and $\bar{z}-\bar{t}(\sigma)=$ $|x-y(\sigma)| e^{-i \psi(x, y(\sigma))}$. Let $\mathbf{n}_{s}$ and $\tau_{s}$ be taken in the point $x^{0}(s) \in \Gamma$. Then for $y(\sigma) \in \Gamma$ and $x \neq y$, the following relationships hold:

$$
\begin{gathered}
\left(\tau_{s}, \nabla_{x}\right) e^{-i \psi(x, y(\sigma))}=i e^{-i \psi(x, y(\sigma))} \frac{\sin (\psi(x, y(\sigma))-\alpha(s))}{|x-y(\sigma)|} \\
\frac{\sin (\psi(x, y(\sigma))-\alpha(s))}{|x-y(\sigma)|}=-\operatorname{Im}\left[\frac{e^{i \alpha(s)}}{z-t}\right]=-\frac{1}{2 i}\left[\frac{e^{i \alpha(s)}}{z-t}-\frac{e^{-i \alpha(s)}}{\bar{z}-\bar{t}}\right], \\
\left(\mathbf{n}_{s}, \nabla_{x}\right) e^{-i \psi(x, y(\sigma))}=i e^{-i \psi(x, y(\sigma))} \frac{\cos (\psi(x, y(\sigma))-\alpha(s))}{|x-y(\sigma)|} \\
\frac{\cos (\psi(x, y(\sigma))-\alpha(s))}{|x-y(\sigma)|}=\operatorname{Re}\left[\frac{e^{i \alpha(s)}}{z-t}\right]=\frac{1}{2}\left[\frac{e^{i \alpha(s)}}{z-t}+\frac{e^{-i \alpha(s)}}{\bar{z}-\bar{t}}\right],
\end{gathered}
$$

where $\alpha(s)$ is the inclination of the tangent $\tau_{s}$ to the $O x_{1}$-axis. To derive $\left(\tau_{s}, \nabla_{x} \mathcal{E}\right)$ and $\left(\mathbf{n}_{x}, \nabla_{x} \mathcal{E}\right)$ we have to take into account the relationship

$$
\nabla_{x} e^{-2 i \psi(x, y(\sigma))}=2 e^{-i \psi(x, y(\sigma))} \nabla_{x} e^{-i \psi(x, y(\sigma))}
$$

and have to use the formulae presented above.

Let $\mu(\sigma)$ be integrable on $\Gamma$. If $x \notin \Gamma$ and $\tau_{s}=(\cos \alpha(s), \sin \alpha(s))$ is taken in the point $x^{0}(s) \in \Gamma$, then

$$
\begin{gathered}
\frac{\partial}{\partial \tau_{s}} \mathcal{E}[\mu](x)=\left(\tau_{s}, \nabla \mathcal{E}\right) \\
=\frac{1}{\pi i} \int_{\Gamma} \mu(\sigma) e^{-2 i \psi(x, y(\sigma))}(-1) \frac{\sin (\psi(x, y(\sigma))-\alpha(s))}{|x-y(\sigma)|} d \sigma \\
=\frac{1}{\pi i} \int_{\Gamma} \mu(\sigma) \frac{\bar{z}-\bar{t}(\sigma)}{z-t(\sigma)} \frac{1}{2 i}\left[\frac{e^{i \alpha(s)}}{z-t(\sigma)}-\frac{e^{-i \alpha(s)}}{\bar{z}-\bar{t}(\sigma)}\right] d \sigma \\
=\frac{1}{\pi i} \frac{1}{2 i}\left[e^{-i \alpha(s)} \int_{\Gamma} \hat{\mu}(t) \frac{d t}{t-z}-e^{i \alpha(s)} \int_{\Gamma} \hat{\mu}(t) \frac{\bar{t}-\bar{z}}{t-z} \frac{d t}{t-z}\right],
\end{gathered}
$$

where $z=\left(x_{1}+i x_{2}\right) \notin \Gamma, \quad t=t(\sigma)=\left(y_{1}(\sigma)+i y_{2}(\sigma)\right) \in \Gamma, \quad d t=t^{\prime}(\sigma) d \sigma=e^{i \alpha(\sigma)} d \sigma$, and $\hat{\mu}(t)=\mu(\sigma) e^{-i \alpha(\sigma)}$. In a similar manner, if $x \notin \Gamma$ and $\mathbf{n}_{s}=(\sin \alpha(s),-\cos \alpha(s))$ is 
taken in the point $x^{0}(s) \in \Gamma$, then

$$
\begin{gathered}
\frac{\partial}{\partial \mathbf{n}_{s}} \mathcal{E}[\mu](x)=\left(\mathbf{n}_{s}, \nabla \mathcal{E}\right) \\
=\frac{1}{\pi i} \int_{\Gamma} \mu(\sigma) e^{-2 i \psi(x, y(\sigma))}(-1) \frac{\cos (\psi(x, y(\sigma))-\alpha(s))}{|x-y(\sigma)|} d \sigma \\
=\frac{1}{\pi i} \int_{\Gamma} \mu(\sigma) \frac{\bar{z}-\bar{t}(\sigma)}{z-t(\sigma)}\left(-\frac{1}{2}\right)\left[\frac{e^{i \alpha(s)}}{z-t(\sigma)}+\frac{e^{-i \alpha(s)}}{\bar{z}-\bar{t}(\sigma)}\right] d \sigma \\
=\frac{1}{2 \pi i}\left[e^{-i \alpha(s)} \int_{\Gamma} \hat{\mu}(t) \frac{d t}{t-z}+e^{i \alpha(s)} \int_{\Gamma} \hat{\mu}(t) \frac{\bar{t}-\bar{z}}{t-z} \frac{d t}{t-z}\right] .
\end{gathered}
$$

Hence, to study the behaviour of the derivatives of $\mathcal{E}[\mu](x)$ we have to study the behaviour of the integral

$$
I[\hat{\mu}](z)=\frac{1}{2 \pi i} \int_{\Gamma} \hat{\mu}(t) \frac{\bar{t}-\bar{z}}{t-z} \frac{d t}{t-z}, \quad z=\left(x_{1}+i x_{2}\right) \notin \Gamma .
$$

Let $\Gamma$ be a smooth open arc. Let $\hat{\mu}(t) \in \mathbb{C}^{0, \beta}(\Gamma), \beta \in(0,1]$. Our goal is to study the behaviour of the integral

$$
J[\hat{\mu}](z)=\frac{1}{2 \pi i} \int_{\Gamma} \frac{\hat{\mu}(t)-\hat{\mu}\left(t_{0}\right)}{t-z} \frac{\bar{t}-\bar{z}}{t-z} d t
$$

if $z \rightarrow t_{0}$, where $t_{0}$ is an arbitrary point of the $\operatorname{arc} \Gamma$. (The case when $t_{0}$ coincides with an end of $\Gamma$ is not excluded.)

Lemma 7. Assume that $\Gamma$ is a smooth open arc and $\hat{\mu}(t) \in \mathbb{C}^{0, \beta}(\Gamma), \beta \in(0,1]$. Let $\theta_{0}$ be an arbitrary nonobtuse angle (i.e., $0<\theta_{0} \leq \pi / 2$ ), and let $z$ approach $t_{0}$ so that the nonobtuse angle between the intercept $t_{0} z$ and the tangent line to $\Gamma$ in the point $t_{0}$ is more or less equal to $\theta_{0}$. Then $J[\hat{\mu}](z)$ tends uniformly (with respect to the position of $t_{0}$ on $\Gamma$ ) to the limit

$$
J[\hat{\mu}]\left(t_{0}\right)=\frac{1}{2 \pi i} \int_{\Gamma} \frac{\hat{\mu}(t)-\hat{\mu}\left(t_{0}\right)}{t-t_{0}} \frac{\bar{t}-\bar{t}_{0}}{t-t_{0}} d t
$$

(independently, whether $z$ approaches $t_{0}$ from the left or from the right from the tangent line).

REMARK. Since $\hat{\mu}(t)$ is a Hölder function on $\Gamma$ and

$$
\left|\frac{\bar{t}-\bar{t}_{0}}{t-t_{0}}\right|=|\exp (-2 i \psi(x, y))|=1
$$

the integral $J[\hat{\mu}]\left(t_{0}\right)$ exists as an improper integral for any point $t_{0} \in \Gamma$; i.e., $t_{0}$ may be an interior point of $\Gamma$, or it may be an end of $\Gamma$.

Proof. By $c, c_{0}, c_{1}$ different constants will be denoted. Note that

$$
\begin{aligned}
& \cos 3 \psi=4 \cos ^{3} \psi-3 \cos \psi, \quad \cos \psi(x, y)=\frac{x_{1}-y_{1}}{|x-y|}, \\
& \sin 3 \psi=3 \sin \psi-4 \sin ^{3} \psi, \quad \sin \psi(x, y)=\frac{x_{2}-y_{2}}{|x-y|} .
\end{aligned}
$$

Therefore, the function $\exp (-3 i \psi(x, y))$ is continuous and infinitely differentiable if $x \neq$ $y$, since it does not have singularities if $x \neq y$. Obviously, $|\exp (-3 i \psi(x, y))|=1$ for 
$x \neq y$. The function $F(x, y)=-|x-y|^{\beta / 4} \exp (-3 i \psi(x, y))$ is continuous for all $x, y \in \mathbb{R}^{2}$ and $F(x, x)=0$. Let $\mathcal{D}$ be a bounded open domain and $\Gamma \subset \mathcal{D}$. According to the Cantor theorem, $F(x, y)$ is uniformly continuous in $(x, y) \in \overline{\mathcal{D}}$. It follows from the definition of uniform continuity that

$$
\left|F(x, y)-F\left(x^{0}, y\right)\right| \leq \varepsilon\left(\left|x-x^{0}\right|\right),
$$

where $\varepsilon\left(\left|x-x^{0}\right|\right)$ does not depend on $y$, and $\varepsilon\left(\left|x-x^{0}\right|\right) \rightarrow 0$ as $x \rightarrow x^{0} \in \Gamma$ uniformly with respect to $x^{0}, y \in \Gamma$.

If $z=x_{1}+i x_{2}, t=y_{1}+i y_{2}$, then

$$
F(x, y)=\hat{F}(z, t)=\frac{\bar{t}-\bar{z}}{t-z} \frac{|t-z|}{t-z}|t-z|^{\beta / 4} .
$$

Inequality (4) can be written in the form

$$
\left|\hat{F}(z, t)-\hat{F}\left(t_{0}, t\right)\right| \leq \varepsilon\left(\left|z-t_{0}\right|\right),
$$

where $\varepsilon\left(\left|z-t_{0}\right|\right)$ does not depend on $t$, and $\varepsilon\left(\left|z-t_{0}\right|\right) \rightarrow 0$ as $z \rightarrow t_{0} \in \Gamma$ uniformly with respect to $t_{0}, t \in \Gamma$.

Consider the difference

$$
J[\hat{\mu}](z)-J[\hat{\mu}]\left(t_{0}\right)=\frac{1}{2 \pi i}\left(J_{1}\left(z, t_{0}\right)+J_{2}\left(z, t_{0}\right)\right),
$$

where

$$
\begin{gathered}
J_{1}\left(z, t_{0}\right)=\int_{\Gamma}\left(\hat{\mu}(t)-\hat{\mu}\left(t_{0}\right)\right)\left(\hat{F}(z, t)-\hat{F}\left(t_{0}, t\right)\right) \frac{d t}{\left|t-t_{0}\right|^{1+\beta / 4}}, \\
J_{2}\left(z, t_{0}\right)=\int_{\Gamma}\left(\hat{\mu}(t)-\hat{\mu}\left(t_{0}\right)\right) \hat{F}(z, t)\left(\frac{1}{|t-z|^{1+\beta / 4}}-\frac{1}{\left|t-t_{0}\right|^{1+\beta / 4}}\right) d t .
\end{gathered}
$$

Using Hölder continuity of the function $\hat{\mu}(t)$ and uniform continuity of the function $\hat{F}(z, t)$, we obtain

$$
\begin{gathered}
\left|J_{1}\left(z, t_{0}\right)\right| \leq \varepsilon\left(\left|z-t_{0}\right|\right) \int_{\Gamma} \frac{c_{1} \cdot\left|t-t_{0}\right|^{\beta}}{\left|t-t_{0}\right|^{1+\beta / 4}}|d t| \\
\leq c_{1} \varepsilon\left(\left|z-t_{0}\right|\right) \int_{\Gamma}\left|\frac{t-t_{0}}{s-s_{0}}\right|^{3 \beta / 4-1}\left|s-s_{0}\right|^{3 \beta / 4-1} d s \leq \operatorname{const} \varepsilon\left(\left|z-t_{0}\right|\right),
\end{gathered}
$$

where $\varepsilon\left(\left|z-t_{0}\right|\right) \rightarrow 0$ as $z \rightarrow t_{0} \in \Gamma$ uniformly with respect to $t_{0} \in \Gamma$. When deriving the latter inequality we used the estimate $\left|\frac{t-t_{0}}{s-s_{0}}\right| \leq$ Const, which follows from [4, Lemma 1], because the arc $\Gamma$ is of class $\mathbb{C}^{1}$.

Note that $|\hat{F}(z, t)| \leq|z-t|^{\beta / 4}$. Since

$$
\begin{gathered}
\left|\hat{\mu}(t)-\hat{\mu}\left(t_{0}\right)\right|^{1 / 4} \leq c\left|t-t_{0}\right|^{\beta / 4}, \\
\left|\hat{\mu}(t)-\hat{\mu}\left(t_{0}\right)\right|=\left|\hat{\mu}(t)-\hat{\mu}\left(t_{0}\right)\right|^{3 / 4} \cdot\left|\hat{\mu}(t)-\hat{\mu}\left(t_{0}\right)\right|^{1 / 4} \leq c\left|\hat{\mu}(t)-\hat{\mu}\left(t_{0}\right)\right|^{3 / 4} \cdot\left|t-t_{0}\right|^{\beta / 4},
\end{gathered}
$$


we obtain

$$
\begin{aligned}
\left|J_{2}\left(z, t_{0}\right)\right| \leq c & \int_{\Gamma}\left|\hat{\mu}(t)-\hat{\mu}\left(t_{0}\right)\right|^{3 / 4}\left|t-t_{0}\right|^{\beta / 4}|t-z|^{\beta / 4}\left|\frac{1}{|t-z|^{1+\beta / 4}}-\frac{1}{\left|t-t_{0}\right|^{1+\beta / 4}}\right||d t| \\
& =c \int_{\Gamma} \frac{\hat{\mu}(t)-\left.\hat{\mu}\left(t_{0}\right)\right|^{3 / 4}}{|t-z|\left|t-t_{0}\right|}|t-z|^{1+\beta / 4}-\left|t-t_{0}\right|^{1+\beta / 4}|| d t \mid .
\end{aligned}
$$

The function $|t-z|^{1+\beta / 4}$ is continuously differentiable on $D$ in $z$ and in $t$. Using the Lagrange formula, we deduce

$$
|| t-\left.z\right|^{1+\beta / 4}-\left|t-t_{0}\right|^{1+\beta / 4}\left|\leq c_{0}\right||t-z|-\left|t-t_{0}\right|\left|\leq c_{0}\right| z-t_{0} \mid,
$$

where $c_{0}=$ const. Therefore,

$$
\left|J_{2}\left(z, t_{0}\right)\right| \leq c \cdot c_{0}\left|z-t_{0}\right| \int_{\Gamma} \frac{\left|\hat{\mu}(t)-\hat{\mu}\left(t_{0}\right)\right|^{3 / 4}}{|t-z|\left|t-t_{0}\right|}|d t| .
$$

An analogous integral has been studied in [1, sect. 15] and in [6, sect. 4.1]. This integral can be represented as a sum of two integrals, one of which is integrated over a "standard arc" [1, sect. 2], while another one is integrated over the remaining part of the contour $\Gamma$. Repeating the arguments from [1, sect. 15] or [6, sect. 4.1], we may estimate each of the integrals. Consequently, we obtain that if $z \rightarrow t_{0}$ in such a way as described in the formulation of the lemma, then $\left|J_{2}\left(z, t_{0}\right)\right| \rightarrow 0$ uniformly with respect to $t_{0} \in \Gamma$. The lemma is proved.

Let $\Gamma$ be an arc of class $\mathbb{C}^{1, \lambda}, \lambda \in(0,1]$. Consider the integral

$$
\begin{gathered}
G(z)=\frac{1}{2 \pi i} \int_{\Gamma} \frac{\bar{t}-\bar{z}}{t-z} \frac{d t}{t-z}=-\frac{1}{2 \pi i} \int_{\Gamma}(\bar{t}-\bar{z}) d \frac{1}{t-z} \\
=-\frac{1}{2 \pi i}\left[\left.\frac{\bar{t}-\bar{z}}{t-z}\right|_{t=t(a)} ^{t=t(b)}-\int_{\Gamma} \frac{d \bar{t}}{t-z}\right] \\
=-\frac{1}{2 \pi i}\left[\exp (-2 i \psi(x, y(b)))-\exp (-2 i \psi(x, y(a)))-\int_{\Gamma} \frac{\exp (-2 i \alpha(\sigma)) d t}{t-z}\right],
\end{gathered}
$$

$t=y_{1}(\sigma)+i y_{2}(\sigma)$. Using Lemma 2(1) and 1, sect. 15], we observe that the function $G(z)$ is continuously extensible to $\Gamma$ from the left and from the right in all points except ends. If $t_{0}=x_{1}(s)+i x_{2}(s)$ is an interior point of $\Gamma$ and $z \rightarrow t_{0} \in \Gamma^{ \pm}$, then, using the Plemelj formulae [1, we derive the limit values of $G(z)$ on $\Gamma^{+}$and $\Gamma^{-}$:

$$
G^{ \pm}\left(t_{0}\right)= \pm \frac{1}{2} \exp (-2 i \alpha(s))+G\left(t_{0}\right)
$$

where

$$
G\left(t_{0}\right)=-\frac{1}{2 \pi i}\left[e^{-2 i \psi(x(s), y(b))}-e^{-2 i \psi(x(s), y(a))}-\int_{\Gamma} \frac{\exp (-2 i \alpha(\sigma)) d t}{t-t_{0}}\right]
$$

is the direct value of $G\left(t_{0}\right)$ on $\Gamma$. It follows from the properties of Cauchy integrals that $G\left(t_{0}\right)$ is continuous on $\Gamma$ in interior points, since the density in $G\left(t_{0}\right)$ is a Hölder 
continuous in $t$ function according to Lemma 2(1). Integrating by parts and using the definition of the singular integral, one can show that

$$
G\left(t_{0}\right)=\frac{1}{2 \pi i} \int_{\Gamma} \frac{\bar{t}-\bar{t}_{0}}{t-t_{0}} \frac{d t}{t-t_{0}} .
$$

This integral is defined if $t_{0}$ is an interior point of $\Gamma$ and is studied in Lemma 6. We summarize the obtained results in the following lemma.

Lemma 8. Let $\Gamma$ be an open arc of class $\mathbb{C}^{1, \lambda}, \lambda \in(0,1]$. Then: 1$)$ the function $G(z)$ is continuously extensible to $\Gamma$ from the left and from the right in all points excluding endpoints; 2) the limit values of $G(z)$ on $\Gamma^{+}$and on $\Gamma^{-}$are continuous in the interior points of $\Gamma$; 3) for limit values of $G(z)$ on $\Gamma^{+}$and $\Gamma^{-}$, the formulae (5) hold.

Using the integrals $J[\hat{\mu}](z)$ and $G(z)$, we represent the integral $I[\hat{\mu}](z)$ in the form

$$
I[\hat{\mu}](z)=\frac{1}{2 \pi i} \int_{\Gamma} \frac{\hat{\mu}(t)}{t-z} \frac{\bar{t}-\bar{z}}{t-z} d t=J[\hat{\mu}](z)+\hat{\mu}\left(t_{0}\right) G(z), \quad z \notin \Gamma
$$

Now we prove the theorem.

Theorem 2. Let $\Gamma$ be an open arc of class $\mathbb{C}^{1, \lambda}, \lambda \in(0,1], \hat{\mu}(t) \in \mathbb{C}_{q}^{\beta}(\Gamma), q \in[0,1)$, $\beta \in(0,1]$. Then: 1$)$ the function $I[\hat{\mu}](z)$ is continuously extensible to $\Gamma$ from the left and from the right everywhere, except endpoints; 2) the limit values of $I[\hat{\mu}](z)$ on $\Gamma^{+}$ and on $\Gamma^{-}$are continuous in the interior points of $\Gamma$; 3) for the limit values of $I[\hat{\mu}](z)$ on $\Gamma^{+}$and on $\Gamma^{-}$the following formulae hold:

$$
I^{ \pm}[\hat{\mu}]\left(t_{0}\right)= \pm \frac{1}{2} \exp (-2 i \alpha(s)) \hat{\mu}\left(t_{0}\right)+I[\hat{\mu}]\left(t_{0}\right),
$$

where $z \rightarrow t_{0} \in \Gamma^{ \pm}, t_{0}=x_{1}(s)+i x_{2}(s)$, and

$$
I[\hat{\mu}]\left(t_{0}\right)=\hat{\mu}\left(t_{0}\right) G\left(t_{0}\right)+J[\hat{\mu}]\left(t_{0}\right)=\frac{1}{2 \pi i} \int_{\Gamma} \frac{\hat{\mu}(t)}{t-t_{0}} \frac{\bar{t}-\bar{t}_{0}}{t-t_{0}} d t
$$

is the direct value of $I[\hat{\mu}](z)$ in the interior point of $t_{0}$ of the $\operatorname{arc} \Gamma$.

REMARK. The singular integral

$$
\frac{1}{2 \pi i} \int_{\Gamma} \frac{\hat{\mu}(t)}{t-t_{0}} \frac{\bar{t}-\bar{t}_{0}}{t-t_{0}} d t
$$

is defined and is continuous in interior points of the arc $\Gamma$ by Lemma 6 . The fact that this integral is equal to $\hat{\mu}\left(t_{0}\right) G\left(t_{0}\right)+J[\hat{\mu}]\left(t_{0}\right)$ can be verified directly, by taking into account formula (6) for $G\left(t_{0}\right)$ and by using the definition of the singular integral. 
Proof. Let $\Gamma^{\prime}$ be an arbitrary part of $\Gamma$ such that the ends of $\Gamma^{\prime}$ are interior points of $\Gamma$. Obviously, it is sufficient to prove parts 1), 2) and 3) of the theorem for the arc $\Gamma^{\prime}$, since for any fixed interior point $t_{0} \in \Gamma$ we always may choose $\Gamma^{\prime}$ such that the small vicinity of $t_{0}$ on $\Gamma$ will consist of interior points of $\Gamma^{\prime}$. Let the arc $\Gamma^{\prime \prime}$ be such a part of $\Gamma$ that the ends of $\Gamma^{\prime \prime}$ are interior points of $\Gamma$ and the ends of $\Gamma^{\prime}$ are interior points of $\Gamma^{\prime \prime}$. Clearly, $\hat{\mu}(t) \in \mathbb{C}^{0, \beta}\left(\Gamma^{\prime \prime}\right)$. We divide the integral over $\Gamma$ in $I[\hat{\mu}](z)$ into the sum of two integrals: one of them is integrated over $\Gamma \backslash \Gamma^{\prime \prime}$, while the other one is integrated over $\Gamma^{\prime \prime}$. The integral over $\Gamma \backslash \Gamma^{\prime \prime}$ is a continuous function of the variable $z$ in the vicinity of points of the $\operatorname{arc} \Gamma^{\prime}$. To prove the theorem it is sufficient to consider the integral over $\Gamma^{\prime \prime}$ in the formula (7) and to take into account properties of the functions $J[\hat{\mu}](z)$ and $G(z)$, which are presented in Lemmas 7, 8. The proof of parts 1) and 2) is based on Lemma 7 and is similar to the proof of the theorem from [1, sect. 15], which uses the result from [1, sect. 9]. Part 3) of the theorem follows from Lemma 7 and from formulae (5) for $G^{ \pm}\left(t_{0}\right)$.

Theorem 3. Let $\Gamma$ be an open $\operatorname{arc}$ of class $\mathbb{C}^{1, \lambda}, \lambda \in(0,1], \mu(s) \in \mathbb{C}_{q}^{\omega}[a, b], \omega \in(0,1]$, $q \in[0,1)$. Then the functions $\frac{\partial}{\partial \tau_{s}} \mathcal{E}[\mu](x)$ and $\frac{\partial}{\partial \mathbf{n}_{s}} \mathcal{E}[\mu](x)$ are continuously extensible to $\Gamma$ from the left and from the right in interior points and their limit values on $\Gamma^{+}$and $\Gamma^{-}$ are given by the formulae

$$
\begin{gathered}
\left(\frac{\partial}{\partial \tau_{s}} \mathcal{E}[\mu](x)\right)^{ \pm}=-i\left[\frac{e^{-i \alpha(s)}}{2 \pi i} \int_{\Gamma} \frac{\hat{\mu}(t) d t}{t-t_{0}}-\frac{e^{i \alpha(s)}}{2 \pi i} \int_{\Gamma} \frac{\hat{\mu}(t)}{t-t_{0}} \frac{\bar{t}-\bar{t}_{0}}{t-t_{0}} d t\right] \\
=\frac{i}{\pi} \int_{\Gamma} \mu(\sigma) e^{-2 i \psi(x(s), y(\sigma))} \frac{\sin (\psi(x(s), y(\sigma))-\alpha(s))}{|x(s)-y(\sigma)|} d \sigma \\
\left(\frac{\partial}{\partial \mathbf{n}_{s}} \mathcal{E}[\mu](x)\right)^{ \pm}=\left[ \pm \mu(s) e^{-2 i \alpha(s)}+\frac{e^{-i \alpha(s)}}{2 \pi i} \int_{\Gamma} \frac{\hat{\mu}(t) d t}{t-t_{0}}+\frac{e^{i \alpha(s)}}{2 \pi i} \int_{\Gamma} \frac{\hat{\mu}(t)}{t-t_{0}} \frac{\bar{t}-\bar{t}_{0}}{t-t_{0}} d t\right] \\
= \pm \mu(s) e^{-2 i \alpha(s)}+\frac{i}{\pi} \int_{\Gamma} \mu(\sigma) e^{-2 i \psi(x(s), y(\sigma))} \frac{\cos (\psi(x(s), y(\sigma))-\alpha(s))}{|x(s)-y(\sigma)|} d \sigma,
\end{gathered}
$$

where $t_{0}=x_{1}(s)+i x_{2}(s), t=y_{1}(\sigma)+i y_{2}(\sigma), \hat{\mu}(t)=\mu(\sigma) e^{-i \alpha(\sigma)}$.

Proof. According to part 2 of Lemma 2, the function $\hat{\mu}(t)=\mu(\sigma) \exp (-i \alpha(\sigma))$ belongs to $\mathbb{C}_{q}^{\beta}(\Gamma)$ in the variable $t$, where $\beta=\min \{\lambda, \omega\}$. From [1, Section 15] and from Theorem 2 it follows that the functions $\frac{\partial}{\partial \tau_{s}} \mathcal{E}[\mu](x)$ and $\frac{\partial}{\partial \mathbf{n}_{s}} \mathcal{E}[\mu](x)$, defined by expressions (2), (3), are continuously extensible to $\Gamma$ from the left and from the right in interior points. Setting $x \rightarrow x(s) \in \Gamma^{ \pm}$(i.e. $z \rightarrow t^{0} \in \Gamma^{ \pm}$) in formulae (2), (3), and using both 
Theorem 2 and the Plemelj formulae [1], we deduce

$$
\begin{gathered}
\left(\frac{\partial}{\partial \tau_{s}} \mathcal{E}[\mu](x)\right)^{ \pm}=-i\left[ \pm \frac{1}{2} \mu(s) e^{-2 i \alpha(s)}+\frac{e^{-i \alpha(s)}}{2 \pi i} \int_{\Gamma} \hat{\mu}(t) \frac{d t}{t-t_{0}}\right. \\
\left.\mp \frac{1}{2} \mu(s) e^{-2 i \alpha(s)}-\hat{\mu}\left[t_{0}\right] G\left(t_{0}\right) e^{i \alpha(s)}-e^{i \alpha(s)} J[\hat{\mu}]\left(t_{0}\right)\right] \\
\left(\frac{\partial}{\partial \tau_{s}} \mathcal{E}[\mu](x)\right)^{ \pm}=-i\left[\frac{e^{-i \alpha(s)}}{2 \pi i} \int_{\Gamma} \frac{\hat{\mu}(t) d t}{t-t_{0}}-\hat{\mu}\left[t_{0}\right] G\left(t_{0}\right) e^{i \alpha(s)}-e^{i \alpha(s)} J[\hat{\mu}]\left(t_{0}\right)\right] \\
=-i\left[\frac{e^{-i \alpha(s)}}{2 \pi i} \int_{\Gamma} \frac{\hat{\mu}(t) d t}{t-t_{0}}-\frac{e^{i \alpha(s)}}{2 \pi i} \int_{\Gamma} \frac{\hat{\mu}(t)}{t-t_{0}} \frac{\bar{t}-\bar{t}_{0}}{t-t_{0}} d t\right] \\
=-i\left[\frac{e^{-i \alpha(s)}}{2 \pi i} \int_{\Gamma} \frac{\mu(\sigma) d \sigma}{t-t_{0}}-\frac{e^{i \alpha(s)}}{2 \pi i} \int_{\Gamma} \frac{\mu(\sigma)}{t-t_{0}} e^{-2 i \psi(x, y(\sigma))} d \sigma\right] \\
=\frac{i}{\pi} \int_{\Gamma} \mu(\sigma) e^{-2 i \psi(x(s), y(\sigma))} \frac{\sin (\psi(x(s), y(\sigma))-\alpha(s))}{|x(s)-y(\sigma)|} d \sigma \\
\left(\frac{\partial}{\partial \mathbf{n}_{s}} \mathcal{E}[\mu](x)\right)^{ \pm}=\left[ \pm \frac{1}{2} \mu(s) e^{-2 i \alpha(s)}+\frac{e^{-i \alpha(s)}}{2 \pi i} \int_{\Gamma} \frac{\hat{\mu}(t) d t}{t-t_{0}}\right. \\
\left. \pm \frac{1}{2} \mu(s) e^{-2 i \alpha(s)}+\frac{e^{i \alpha(s)}}{2 \pi i} \int_{\Gamma} \frac{\hat{\mu}(t)}{t-t_{0}} \frac{\bar{t}-\bar{t}_{0}}{t-t_{0}} d t\right] \\
=\left[ \pm \mu(s) e^{-2 i \alpha(s)}+\frac{e^{-i \alpha(s)}}{2 \pi i} \int_{\Gamma} \frac{\hat{\mu}(t) d t}{t-t_{0}}+\frac{e^{i \alpha(s)}}{2 \pi i} \int_{\Gamma} \frac{\hat{\mu}(t)}{t-t_{0}} \frac{\bar{t}-\bar{t}_{0}}{t-t_{0}} d t\right] \\
= \pm \mu(s) e^{-2 i \alpha(s)}-\frac{e^{-i \alpha(s)}}{2 \pi i} \int_{\Gamma} e^{-2 i \psi(x(s), y(\sigma))} \frac{\mu(\sigma)}{|x(s)-y(\sigma)|} e^{i \psi(x(s), y(\sigma))} d \sigma \\
-\frac{e^{i \alpha(s)}}{2 \pi i} \int_{\Gamma} e^{-2 i \psi(x(s), y(\sigma))} \frac{\mu(\sigma)}{|x(s)-y(\sigma)|} e^{-i \psi(x(s), y(\sigma))} d \sigma \\
=\mu(s) e^{-2 i \alpha(s)}-\frac{1}{\pi i} \int_{\Gamma} \mu(\sigma) e^{-2 i \psi(x(s), y(\sigma))} \frac{\cos (\psi(x(s), y(\sigma))-\alpha(s))}{|x(s)-y(\sigma)|} d \sigma \\
\end{gathered}
$$

where $t_{0}=x_{1}(s)+i x_{2}(s), t=y_{1}(\sigma)+i y_{2}(\sigma)$ and $\hat{\mu}(t)=\mu(\sigma) e^{-i \alpha(\sigma)}$. The theorem is proved.

Corollary 1. If the conditions of Theorem 3 hold, then

$$
\nabla \mathcal{C}[\mu](x), \quad \nabla \mathcal{S}[\mu](x) \in \mathbb{C}^{0}\left(\overline{\mathbb{R}^{2} \backslash \Gamma} \backslash X_{\Gamma}\right),
$$

where $X_{\Gamma}=\{x(a) \cup x(b)\}$.

REMARK. Each function of the class $\mathbb{C}^{0}\left(\overline{\mathbb{R}^{2} \backslash \Gamma} \backslash X_{\Gamma}\right)$ is continuous in $\mathbb{R}^{2} \backslash \Gamma$ and is continuously extensible to $\Gamma \backslash X_{\Gamma}$ from the left and right, but the limiting values of such a function on $\Gamma \backslash X_{\Gamma}$ can be different from the left and right; i.e., the function may have a jump on $\Gamma \backslash X_{\Gamma}$.

5. Estimates of $I[\hat{\mu}](z)$ at the ends of the $\operatorname{arc} \Gamma$. Now we will study the behaviour of $\nabla \mathcal{E}[\mu](x)$ in the neighbourhood of the ends of the integration line. For this purpose we have to study the behaviour of the integral $I[\mu](z)$ from $(7)$ near the ends of the $\operatorname{arc} \Gamma$. 
Lemma 9. Let $\Gamma$ be a smooth open $\operatorname{arc} \hat{\mu}(t) \in \mathbb{C}^{0, \beta}(\Gamma)$ and $\hat{\mu}(t(a))=0($ or $\hat{\mu}(t(b))=0)$. Then the function $I[\hat{\mu}](z)$ is continuously extensible at the end $t(a)$ (or $t(b)$ ) of the $\operatorname{arc} \Gamma$.

The proof will be given for $t(a)$ (for $t(b)$ it is analogous). We extend $\Gamma$ through the end $t(a)$ smoothly and set $\hat{\mu}(t) \equiv 0$ on the added part. We denote the new extended arc by $\Gamma^{\prime}$. Then $\hat{\mu}(t) \equiv 0$ on $\Gamma^{\prime} \backslash \Gamma$ and

$$
I[\mu](z)=\frac{1}{2 \pi i} \int_{\Gamma^{\prime}} \frac{\mu(t)}{t-z} \frac{\bar{t}-\bar{z}}{t-z} d t .
$$

In accordance with the properties of Hölder functions [1, sect. 5.1], $\hat{\mu}(t) \in \mathbb{C}^{0, \beta}\left(\Gamma^{\prime}\right)$, and the point $t(a)$ is an interior point for the arc $\Gamma^{\prime}$. Using Theorem 2 in the point $t(a)$, we deduce:

$$
I^{+}[\hat{\mu}]\left(t_{0}(a)\right)=I^{-}[\hat{\mu}]\left(t_{0}(a)\right)=\frac{1}{2 \pi i} \int_{\Gamma} \frac{\hat{\mu}(t)}{t-t_{0}(a)} \frac{\bar{t}-\bar{t}_{0}(a)}{t-t_{0}(a)} d t .
$$

The lemma is proved.

Lemma 10. Let $\Gamma$ be a smooth open arc, $\hat{\mu}(t) \in \mathbb{C}_{q}^{\beta}(\Gamma), \quad \beta \in(0,1], q \in[0,1)$, and let $\gamma$ be an arbitrary number such that $q<\gamma<1$. Then for points $z$, located in the neighbourhood of the end $t(d)$ of the $\operatorname{arc} \Gamma$ (where $d=a$ or $d=b$ ) the following estimate holds:

$$
|I[\hat{\mu}](z)| \leq \frac{\text { const }}{|z-t(d)|^{\gamma}} .
$$

The proof of the lemma will be given for $d=a$, because for $d=b$ the proof is similar. Since $\hat{\mu}(t) \in \mathbb{C}_{q}^{\beta}(\Gamma)$, we observe that $\hat{\mu}(t)$ can be expressed in the form

$$
\hat{\mu}(t)=\frac{\hat{\mu}_{0}(t)}{|t-t(a)|^{q}|t-t(b)|^{q}},
$$

where $\hat{\mu}_{0}(t) \in \mathbb{C}^{0, \beta}(\Gamma)$. Let the arc $l \subset \Gamma$ be such that its ends are the point $t(a)$ and some interior point of $\Gamma$. The integral $I[\mu](z)$ can be represented in the form of a sum of two integrals; one of them is over $l$, while the other one is over $\Gamma \backslash l$. Obviously, the integral over $\Gamma \backslash l$ is continuous and so is bounded if the point $z$ is placed in the vicinity of the point $t(a)$. Hence, this integral satisfies the estimate required in the lemma.

Now we will prove the estimate for the integral over $l$ :

$$
I_{0}(z)=\frac{1}{2 \pi i} \int_{l} \frac{\hat{\mu}_{*}(t)}{|t-t(a)|^{q}} \frac{\bar{t}-\bar{z}}{t-z} \frac{d t}{t-z},
$$

where $\hat{\mu}_{*}(t)=\hat{\mu}_{0}(t)|t-t(b)|^{-q} \in \mathbb{C}^{0, \beta}(l)$, because $t(b) \notin l$. Consider

$$
\begin{gathered}
|z-t(a)|^{\gamma} I_{0}(z)=\frac{1}{2 \pi i} \int_{l} \frac{|z-t(a)|^{\gamma}-|t-t(a)|^{\gamma}}{|t-t(a)|^{q}(t-z)} \cdot \frac{\bar{t}-\bar{z}}{t-z} \cdot \hat{\mu}_{*}(t) d t \\
+\frac{1}{2 \pi i} \int_{l} \frac{|t-t(a)|^{\gamma-q}}{t-z} \cdot \frac{\bar{t}-\bar{z}}{t-z} \cdot \hat{\mu}_{*}(t) d t .
\end{gathered}
$$

Since the function $|t-t(a)|^{\gamma-q} \hat{\mu}_{*}(t)$ is Hölder continuous on $l$ and is equal to zero as $t=t(a)$, we observe that the second integral is bounded as $z \rightarrow t(a)$ by Lemma 9 . 
The first integral in the right part we denote by $I_{1}$ and consider this integral. Since ||$z-\left.t(a)\right|^{\gamma}-|t-t(a)|^{\gamma}|\leq| z-\left.t\right|^{\gamma}$ according to [1, sect. 5], we have

$$
\left|I_{1}\right| \leq \frac{\mathrm{const}}{2 \pi} \int_{l} \frac{1}{|t-t(a)|^{q}} \frac{1}{|t-z|^{1-\gamma}}|d t|,
$$

where we took into account that $\left|\hat{\mu}_{*}(t)\right| \leq$ const on $l$ and $\left|\frac{\bar{z}-\bar{t}}{z-t}\right|=1$. In the same way as in [1, sect. 23.1], we obtain that $\left|I_{1}\right| \leq$ const, since $q+1-\gamma<1$. Therefore,

$$
|z-t(a)|^{\gamma}\left|I_{0}(z)\right| \leq \text { const }
$$

and so

$$
\left|I_{0}(z)\right| \leq \frac{\text { const }}{|z-t(a)|^{\gamma}}
$$

From here we obtain the statement of the lemma.

6. Asymptotics of $I[\hat{\mu}(t)](z)$ at the ends of the $\operatorname{arc} \Gamma$. Let us study the behaviour of the integral

$$
\begin{gathered}
\mathcal{I}(z)=\frac{1}{2 \pi i} \int_{\Gamma} \frac{1}{(t-D)^{q}} \frac{\bar{t}-\bar{z}}{t-z} \frac{d t}{t-z}=\mathcal{I}_{1}(z)-(\bar{z}-\bar{D}) \frac{d}{d z} \mathcal{I}_{2}(z), \\
\mathcal{I}_{1}(z)=\frac{1}{2 \pi i} \int_{\Gamma} \frac{\bar{t}-\bar{D}}{(t-D)^{q}} \frac{d t}{(t-z)^{2}}, \quad \mathcal{I}_{2}(z)=\frac{1}{2 \pi i} \int_{\Gamma} \frac{1}{(t-D)^{q}} \frac{d t}{(t-z)},
\end{gathered}
$$

where $D=t(d)$ and $d=a$ or $d=b$. In other words, $D=t(a)$ or $D=t(b)$. It is shown in [1, sect. 23.2] that if $z$ is placed near $D$ and $z \notin \Gamma$, then

$$
\mathcal{I}_{2}(z)=\delta(D) \frac{e^{i \pi q \delta(D)}}{2 i \sin (\pi q)} \frac{1}{(z-D)^{q}}+\Omega^{D}(z)
$$

where

$$
\delta(D)=\left\{\begin{array}{l}
1, \text { if } D=t(a), \text { i.e. } d=a, \\
-1, \text { if } D=t(b), \text { i.e } d=b .
\end{array}\right.
$$

The function $\Omega^{D}(z)$ is analytic in the neighbourhood of the point $z=D$. By $(z-D)^{q}$ we mean the single-valued branch of this function in the neighbourhood of the point $D$, cut along $\Gamma$. It is assumed that the function $(z-D)^{q}$ takes the value $(t-D)^{q}$ (see (8)) on the left side of $\Gamma$ (i.e. on $\Gamma^{+}$). Differentiating (9), we obtain

$$
-(\bar{z}-\bar{D}) \frac{d}{d z} \mathcal{I}_{2}(z)=q \delta(D) \frac{e^{i \pi q \delta(D)}}{2 i \sin (\pi q)} \frac{\bar{z}-\bar{D}}{z-D} \frac{1}{(z-D)^{q}}-(\bar{z}-\bar{D}) \Omega_{1}^{D}(z),
$$

where $\Omega_{1}^{D}(z)=\frac{d}{d z} \Omega^{D}(z)$ is an analytic function in the neighbourhood of the point $D$. Assuming that $t=t(\sigma)=\left(y_{1}(\sigma)+i y_{2}(\sigma)\right) \in \Gamma$ and integrating $\mathcal{I}_{1}(z)$ by parts, we obtain

$$
\begin{gathered}
\mathcal{I}_{1}(z)=-\frac{1}{2 \pi i} \int_{\Gamma} \frac{\bar{t}-\bar{D}}{(t-D)^{q}} d \frac{1}{t-z}=\frac{1}{2 \pi i} \int_{\Gamma} \frac{1}{t-z} d \frac{\bar{t}-\bar{D}}{(t-D)^{q}}+\Omega_{0}^{D}(z) \\
=\frac{1}{2 \pi i} \int_{\Gamma} \frac{\varphi_{D}(\sigma)}{t-z} \frac{1}{(t-D)^{q}} d \sigma+\Omega_{0}^{D}(z)=\frac{1}{2 \pi i} \int_{\Gamma} \frac{\hat{\varphi}_{D}(t)}{t-z} \frac{1}{(t-D)^{q}} d t+\Omega_{0}^{D}(z),
\end{gathered}
$$


where $\Omega_{0}^{D}(z)$ is an analytic function in the neighbourhood of the point $D$; more precisely,

$$
\begin{aligned}
& \Omega_{0}^{D}(z)=-\frac{1}{2 \pi i} \frac{\bar{t}(b)-\bar{t}(a)}{(t(b)-t(a))^{q}} \frac{1}{t(b)-z}, \quad \text { if } D=t(a), \\
& \Omega_{0}^{D}(z)=\frac{1}{2 \pi i} \frac{\bar{t}(a)-\bar{t}(b)}{(t(a)-t(b))^{q}} \frac{1}{t(a)-z}, \quad \text { if } D=t(b) .
\end{aligned}
$$

Since

$$
\frac{d}{d \sigma} \frac{\bar{t}(\sigma)-\bar{D}}{(t(\sigma)-D)^{q}}=\frac{\bar{t}^{\prime}(\sigma)(t(\sigma)-D)^{q}-q(t(\sigma)-D)^{q-1}(\bar{t}(\sigma)-\bar{D}) t^{\prime}(\sigma)}{(t(\sigma)-D)^{2 q}}
$$

and since $t^{\prime}(\sigma)=e^{i \alpha(\sigma)}, \bar{t}^{\prime}(\sigma)=e^{-i \alpha(\sigma)}$, we have

$$
\begin{aligned}
\varphi_{D}(\sigma) & =(t(\sigma)-D)^{q} \frac{d}{d \sigma} \frac{\bar{t}(\sigma)-\bar{D}}{(t(\sigma)-D)^{q}}=e^{-i \alpha(\sigma)}-q \frac{\bar{t}(\sigma)-\bar{D}}{t(\sigma)-D} e^{i \alpha(\sigma)} \\
& =e^{-i \alpha(\sigma)}-q e^{-2 i \psi(x(d), y(\sigma))} e^{i \alpha(\sigma)} \\
\hat{\varphi}_{D}(t) & =\hat{\varphi}_{D}(t(\sigma))=\varphi_{D}(\sigma) e^{-i \alpha(\sigma)}
\end{aligned}
$$

Here

$$
x(d)=\left\{\begin{array}{c}
x(a), \text { if } D=t(a), \text { i.e. } d=a, \\
x(b), \text { if } D=t(b), \text { i.e. } d=b .
\end{array}\right.
$$

If $\Gamma$ is an open arc of class $\mathbb{C}^{1, \lambda}$, then, according to Theorem 4(1):

$$
\varphi_{D}(\sigma) \in \mathbb{C}^{0, \lambda}[a, b] .
$$

Hence, by Lemma 2(1), $\hat{\varphi}_{D}(t) \in \mathbb{C}^{0, \lambda}(\Gamma)$. Set $e^{-i \alpha(a)}=e^{-i \alpha(a+0)}, e^{-i \alpha(b)}=e^{-i \alpha(b-0)}$. Obviously, if $\sigma \in[a, b]$, then

$$
\lim _{\sigma \rightarrow a} e^{-2 i \psi(x(a), y(\sigma))}=e^{-2 i \alpha(a)}, \quad \lim _{\sigma \rightarrow b} e^{-2 i \psi(x(b), y(\sigma))}=e^{-2 i \alpha(b)} ;
$$

consequently, if $t \in \Gamma$, then

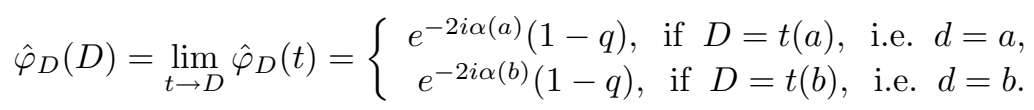

We write $\mathcal{I}_{1}(z)$ in the form

$$
\mathcal{I}_{1}(z)=\frac{\hat{\varphi}_{D}(D)}{2 \pi i} \int_{\Gamma} \frac{1}{(t-D)^{q}} \frac{d t}{t-z}+\frac{1}{2 \pi i} \int_{\Gamma} \frac{\hat{\varphi}_{D}(t)-\hat{\varphi}_{D}(D)}{(t-D)^{q}} \frac{d t}{t-z}+\Omega_{0}^{D}(z) .
$$

The second integral is an analytic function in the $z$ variable in the neighbourhood of the point $D$, cut along $\Gamma$. If $\lambda>q$, then the second integral is bounded (and continuous) when $z \rightarrow D$ in accordance with [1, sect. 18.1]. If $0<\lambda \leq q<1$, then for any $q_{0} \in(0, \lambda)$ :

$$
\frac{\hat{\varphi}_{D}(t)-\hat{\varphi}_{D}(D)}{(t-D)^{q_{0}}} \in \mathbb{C}^{0, \lambda-q_{0}}(\Gamma)
$$

(see [1, sect. 18.3]). Therefore, it follows from [1, sect. 22.2] that if $z$ is placed near $D$ and $z \notin \Gamma$, then, for the second integral in (14), the following estimate holds:

$$
\begin{aligned}
\left|\int_{\Gamma} \frac{\hat{\varphi}_{D}(t)-\hat{\varphi}_{D}(D)}{(t-D)^{q}} \frac{d t}{t-z}\right| & =\left|\int_{\Gamma} \frac{\hat{\varphi}_{D}(t)-\hat{\varphi}_{D}(D)}{(t-D)^{q_{0}}} \frac{1}{(t-D)^{q-q_{0}}} \frac{d t}{t-z}\right| \\
& \leq \frac{\text { const }}{|z-D|^{q-q_{0}}} .
\end{aligned}
$$


If $z$ is placed near $D$ and $z \notin \Gamma$, then applying the formula (9) to the first integral in the right-hand side of (14), we obtain

$$
\mathcal{I}_{1}(z)=\delta(D) \hat{\varphi}_{D}(D) \frac{e^{i \pi q \delta(D)}}{2 i \sin (\pi q)} \frac{1}{(z-D)^{q}}+\Omega_{2}^{D}(z) .
$$

The designation $\hat{\varphi}_{D}(D)$ is defined in (13). By $\Omega_{2}^{D}(z)$ we denote the sum of three functions, namely, the second term in (14), the function $\Omega_{0}^{D}(z)$ from (14) and the function $\Omega^{D}(z)$ from (9). Recall that $\Omega^{D}(z)$ and $\Omega_{0}^{D}(z)$ are analytic functions in the neighbourhood of the point $D$. Thus, the function $\Omega_{2}^{D}(z)$ is analytic in the neighbourhood of the point $D$, cut along $\Gamma$. It follows from the expression for $\Omega_{2}^{D}(z)$ that $\Omega_{2}^{D}(z)$ is continuous when $z \rightarrow D$ if $1 \geq \lambda>q \geq 0$. If $0<\lambda \leq q<1$, then the estimate

$$
\left|\Omega_{2}^{D}(z)\right| \leq \frac{\text { const }}{|z-D|^{q-q_{0}}}
$$

holds for any $q_{0} \in(0, \lambda)$ and for any $z$, placed outside $\Gamma$ near $D$. From relationships (8), (11), (15), we obtain that, for $z$ lying near $D$ outside $\Gamma$, the following formula holds:

$$
\mathcal{I}(z)=\left(\hat{\varphi}_{D}(D)+q \frac{\bar{z}-\bar{D}}{z-D}\right) \delta(D) \frac{e^{i \pi q \delta(D)}}{2 i \sin (\pi q)} \frac{1}{(z-D)^{q}}+\Omega_{2}^{D}(z)-(\bar{z}-\bar{D}) \Omega_{1}^{D}(z) .
$$

The constants $\hat{\varphi}_{D}(D)$ and $\delta(D)$ have been introduced in (10) and in (13). The properties of the functions $\Omega_{1}^{D}(z)$ and $\Omega_{2}^{D}(z)$ are described above. Note that $\frac{\bar{z}-\bar{D}}{z-D}=e^{-2 i \psi(x, x(d))}$, where the constant $x(d)$ is given by the formula (12). Let $\hat{\mu}(t)=\frac{\hat{\nu}(t)}{(t-D)^{q}}, \quad \hat{\nu}(t) \in$ $\mathbb{C}^{0, \beta}(\Gamma), \beta \in(0,1]$. Then

$$
I[\hat{\mu}](z)=\hat{\nu}(D) \mathcal{I}(z)+\Theta(z),
$$

where $I[\hat{\mu}](z), \mathcal{I}(z)$ are defined in $(7),(8)$, and

$$
\Theta(z)=\frac{1}{2 \pi i} \int_{\Gamma} \frac{\hat{\nu}(t)-\hat{\nu}(D)}{(t-D)^{q}} \frac{\bar{t}-\bar{z}}{t-z} \frac{d t}{t-z} .
$$

If $\beta>q$, then according to [1, sect. 6.3],

$$
\frac{\hat{\nu}(t)-\hat{\nu}(D)}{(t-D)^{q}} \in \mathbb{C}^{0, \beta-q}(\Gamma)
$$

and by Lemma 9 the function $\Theta(z)$ is continuous when $z \rightarrow D$. If $0<\beta \leq q<1$, then for any $q_{1} \in(q-\beta, q)$ we choose $q_{0} \in\left(q-q_{1}, \beta\right)$ and rewrite $\Theta(z)$ in the form

$$
\Theta(z)=\frac{1}{2 \pi i} \int_{\Gamma} \frac{\hat{\nu}(t)-\hat{\nu}(D)}{(t-D)^{q_{0}}} \frac{1}{(t-D)^{q-q_{0}}} \frac{\bar{t}-\bar{z}}{t-z} \frac{d t}{t-z} .
$$

Clearly,

$$
\frac{\hat{\nu}(t)-\hat{\nu}(D)}{(t-D)^{q_{0}}} \in \mathbb{C}^{0, \beta-q_{0}}(\Gamma),
$$

and $q-q_{0}<q_{1}<q$. Therefore, if $0<\beta \leq q<1$, then from Lemma 10 it follows that for $z$ lying near $D$ outside $\Gamma$ the estimate

$$
|\Theta(z)| \leq \frac{\text { const }}{|z-D|^{q_{1}}}
$$

holds for any $q_{1} \in(q-\beta, q)$. Let us formulate the obtained result in the form of a lemma. 
Lemma 11. Let $\Gamma$ be an open arc of class $\mathbb{C}^{1, \lambda}, \lambda \in(0,1]$. Let $\hat{\mu}(t)=\frac{\hat{\nu}(t)}{(t-D)^{q}}$, where $q \in(0,1), \quad \hat{\nu}(t) \in \mathbb{C}^{0, \beta}(\Gamma), \quad \beta \in(0,1]$ and $D=t(a)$ or $D=t(b)$. If $z$ lies in the neighbourhood of the point $D$ and $z \notin \Gamma$, then the integral $I[\mu](z)$ can be represented in the form (17), where $\mathcal{I}(z)$ is given by formula (16).

Corollary 2. Let the conditions of Lemma 11 hold. If $z$ lies near $D$ and $z \notin \Gamma$, then the following estimate holds:

$$
|I[\hat{\mu}](z)| \leq \frac{\text { const }}{|z-D|^{q}}
$$

7. Singularities of derivatives of $\mathcal{E}[\mu](x)$ at the ends of the arc $\Gamma$. Let $x=$ $\left(x_{1}, x_{2}\right) \notin \Gamma, y(\sigma)=\left(y_{1}(\sigma), y_{2}(\sigma)\right) \in \Gamma$. Since

$$
\nabla_{x} e^{-i \psi(x, y(\sigma))}=\left(i e^{-i \psi(x, y(\sigma))} \frac{\sin \psi(x, y(\sigma))}{|x-y(\sigma)|},-i e^{-i \psi(x, y(\sigma))} \frac{\cos \psi(x, y(\sigma))}{|x-y(\sigma)|}\right),
$$

we have

$$
\begin{gathered}
\frac{\partial}{\partial x_{1}} \mathcal{E}[\mu](x)=-\frac{1}{\pi i} \int_{\Gamma} \mu(\sigma) e^{-2 i \psi(x, y(\sigma))} \frac{\sin \psi(x, y(\sigma))}{|x-y(\sigma)|} d \sigma \\
=-\frac{1}{2 \pi} \int_{\Gamma} \hat{\mu}(t)\left[\frac{1}{t-z}-\frac{\bar{t}-\bar{z}}{t-z} \frac{1}{t-z}\right] d t, \\
\frac{\partial}{\partial x_{2}} \mathcal{E}[\mu](x)=\frac{1}{\pi i} \int_{\Gamma} \mu(\sigma) e^{-2 i \psi(x, y(\sigma))} \frac{\cos \psi(x, y(\sigma))}{|x-y(\sigma)|} d \sigma \\
=-\frac{1}{2 \pi i} \int_{\Gamma} \hat{\mu}(t)\left[\frac{1}{t-z}+\frac{\bar{t}-\bar{z}}{t-z} \frac{1}{t-z}\right] d t,
\end{gathered}
$$

where $t=\left(y_{1}(\sigma)+i y_{2}(\sigma)\right) \in \Gamma, \quad z=\left(x_{1}+i x_{2}\right) \notin \Gamma$, and $\hat{\mu}(t)=\mu(\sigma) e^{-i \alpha(\sigma)}$.

Let $\Gamma$ be an open arc of class $\mathbb{C}^{1, \lambda}, \lambda \in(0,1]$. Let $\mu(\sigma)=\frac{\mu_{1}(\sigma)}{|\sigma-d|^{q}}$, where $d=a$ or $d=b, q \in(0,1)$ and $\mu_{1}(\sigma) \in \mathbb{C}^{0, \omega}[a, b], \omega \in(0,1]$.

From the results of Lemma 1 in [4], it follows that

$$
\frac{t(\sigma)-t(d)}{|\sigma-d|} \in \mathbb{C}^{0, \lambda}[a, b]
$$

The function $\xi^{q}$ on the complex plane of the variable $\xi$ belongs to the class $\mathbb{C}^{1}$ in any simply connected bounded domain, not containing the point $\xi=0$ (by $\xi^{q}$ we mean the fixed branch, which is continuous in this domain). Since $\frac{t(\sigma)-t(d)}{|\sigma-d|} \neq 0$ for each $\sigma \in[a, b]$, we have

$$
\left(\frac{t(\sigma)-t(d)}{|\sigma-d|}\right)^{q} \in \mathbb{C}^{0, \lambda}[a, b]
$$

Set $D=t(d)$. Then $\mu(\sigma)=\left(\frac{1}{t-D}\right)^{q} \nu(\sigma)$, where

$$
\nu(\sigma)=\left(\frac{t(\sigma)-t(d)}{|\sigma-d|}\right)^{q} \mu_{1}(\sigma) \in \mathbb{C}^{0, \beta}[a, b], \quad \beta=\min \{\omega, \lambda\} .
$$


Set $\hat{\nu}(t)=\hat{\nu}(t(\sigma))=\nu(\sigma) e^{-i \alpha(\sigma)}$. Then by Lemma $2(1), \hat{\nu}(t) \in \mathbb{C}^{0, \beta}(\Gamma)$. Since $\hat{\mu}(t)=$ $\hat{\mu}(t(\sigma))=\mu(\sigma) e^{-i \alpha(\sigma)}$, we obtain

$$
\hat{\mu}(t)=\mu(\sigma) e^{-i \alpha(\sigma)}=\frac{\hat{\nu}(t)}{(t-D)^{q}} .
$$

Substitute $\hat{\mu}(t)$ into (18), (19). From the results of [1, sect. 22] and from Lemma 11, we obtain

Theorem 4. Let $\Gamma$ be an open arc of class $\mathbb{C}^{1, \lambda}, \lambda \in(0,1]$;

$$
\mu(\sigma)=\frac{\mu_{1}(\sigma)}{|\sigma-d|^{q}}, \quad q \in(0,1) ; \quad \mu_{1}(\sigma) \in \mathbb{C}^{0, \omega}[a, b], \quad \omega \in(0,1] ;
$$

$d=a$ or $d=b$. Let

$$
\hat{\nu}(t)=\hat{\nu}(t(\sigma))=\left(\frac{t(\sigma)-t(d)}{|\sigma-d|}\right)^{q} \mu_{1}(\sigma) e^{-i \alpha(\sigma)} .
$$

Then $\hat{\nu}(t) \in \mathbb{C}^{0, \beta}(\Gamma)$, where $\beta=\min \{\omega, \lambda\}$ and for points $z=x_{1}+i x_{2}$, lying near $D=t(d)$ outside $\Gamma$, the following asymptotic formula holds:

$$
\begin{gathered}
\frac{\partial}{\partial x_{j}} \mathcal{E}[\mu](x)=-\delta(D) i^{2-j} \frac{e^{\delta(D) q \pi i}}{2 i \sin (\pi q)} \frac{\hat{\nu}(D)}{(z-D)^{q}} \\
\times\left[1+(-1)^{j}\left(\hat{\varphi}_{D}(D)+q \frac{\bar{z}-\bar{D}}{z-D}\right)\right]+\Theta_{0}(z), \quad j=1,2 .
\end{gathered}
$$

Here $\Theta_{0}(z)$ is continuous when $z \rightarrow D$ if $\beta>q$. If $0<\beta \leq q<1$, then for $z$, lying near $D=t(d)$ outside $\Gamma$, the following estimate holds:

$$
\left|\Theta_{0}(z)\right| \leq \frac{\text { const }}{|z-D|^{q_{1}}},
$$

where $q_{1}$ is an arbitrary number, such that $q_{1} \in(q-\beta, q)$. The parameters $\delta(D)$ and $\hat{\varphi}_{D}(D)$ are defined in $(10),(13)$. By $(z-D)^{q}$ we denote the branch that is analytic in the neighbourhood of $D$ cut along $\Gamma$ and that takes the value $(t-D)^{q}$ on $\Gamma^{+}$(see (20), (21)). In addition,

$$
\hat{\nu}(D)=\lim _{\sigma \rightarrow d} \hat{\nu}(t(\sigma)), \quad \text { where } \quad \sigma \in(a, b) .
$$

Corollary 3. Let $\Gamma$ be an open arc of class $\mathbb{C}^{1, \lambda}, \quad \lambda \in(0,1]$ and $\mu(s) \in \mathbb{C}_{q}^{\omega}[a, b]$, $\omega \in(0,1], q \in(0,1)$. Then for points $x$, lying near $x(d)$ outside $\Gamma(d=a$ or $d=b)$ the following estimate holds:

$$
\left|\frac{\partial}{\partial x_{j}} \mathcal{E}[\mu](x)\right| \leq \frac{\text { const }}{|x-x(d)|^{q}}, \quad j=1,2 .
$$

Let $\Gamma$ be an open arc of class $\mathbb{C}^{1, \lambda}, \lambda \in(0,1]$. If $\mu(\sigma) \in \mathbb{C}^{0, \omega_{1}}[a, b], \omega_{1} \in(0,1]$, then the function $\mu(\sigma)$ can be represented in the form

$$
\mu(\sigma)=\frac{\mu_{1}(\sigma)}{|\sigma-d|^{q}},
$$

for any $q \in(0,1)$, where

$$
\mu_{1}(\sigma)=\mu(\sigma)|\sigma-d|^{q} \in \mathbb{C}^{0, \omega}[a, b], \quad \omega=\min \left\{q, \omega_{1}\right\},
$$


$d=a$ or $d=b$. It follows from Theorem 4 that in this case,

$$
\left|\frac{\partial}{\partial x_{j}} \mathcal{E}[\mu](x)\right| \leq \frac{\text { const }}{|x-x(d)|^{q}}, \quad j=1,2,
$$

for any $q \in(0,1)$ and for all $x$ lying near $x(d)$ outside $\Gamma$. Hence, the following statement holds.

Corollary 4. Let $\Gamma$ be an open arc of class $\mathbb{C}^{1, \lambda}, \quad \lambda \in(0,1]$ and $\mu(\sigma) \in \mathbb{C}^{0, \omega_{1}}[a, b]$, $\omega_{1} \in(0,1]$. Then estimate $(22)$ holds for any $q \in(0,1)$ and for all points $x$ lying near the point $x(d)$ outside $\Gamma(d=a$ or $d=b)$.

8. Pressure potential for Stokes equations. The goal of this section is to study properties of a pressure potential for the Stokes equations [2]:

$$
P\left[\mu_{1}, \mu_{2}\right](x)=P_{c}\left[\mu_{1}\right](x)+P_{s}\left[\mu_{2}\right](x),
$$

where

$$
\begin{gathered}
P_{c}\left[\mu_{1}\right](x)=\frac{1}{2 \pi} \int_{\Gamma} \mu_{1}(\sigma) \frac{\cos (\psi(x, y(\sigma)))}{|x-y(\sigma)|} d \sigma, \\
P_{s}\left[\mu_{2}\right](x)=\frac{1}{2 \pi} \int_{\Gamma} \mu_{2}(\sigma) \frac{\sin (\psi(x, y(\sigma)))}{|x-y(\sigma)|} d \sigma, \\
\cos \psi(x, y(\sigma))=\frac{x_{1}-y_{1}(\sigma)}{|x-y(\sigma)|}, \quad \sin \psi(x, y(\sigma))=\frac{x_{2}-y_{2}(\sigma)}{|x-y(\sigma)|}, \\
y(\sigma)=\left(y_{1}(\sigma), y_{2}(\sigma)\right) \in \Gamma, \quad|x-y(\sigma)|=\sqrt{\left(x_{1}-y_{1}(\sigma)\right)^{2}+\left(x_{2}-y_{2}(\sigma)\right)^{2}} .
\end{gathered}
$$

Densities $\mu_{1}(s), \mu_{2}(s)$ are assumed to be integrable and real-valued. Let $z=x_{1}+i x_{2}$, $t=t(\sigma)=\left(y_{1}(\sigma)+i y_{2}(\sigma)\right) \in \Gamma$. Then $\quad d t=t^{\prime}(\sigma) d \sigma=e^{i \alpha(\sigma)} d \sigma$ and $z-t(\sigma)=$ $|x-y(\sigma)| e^{i \psi(x, y(\sigma))}$. Assuming that $\mu(s)$ is a real integrable density, we consider the complex potential

$$
\begin{gathered}
P_{E}[\mu](x)=P_{c}[\mu](x)-i P_{s}[\mu](x) \\
=\frac{1}{2 \pi} \int_{\Gamma} \mu(\sigma) \frac{e^{-i \psi(x, y(\sigma))}}{|x-y(\sigma)|} d \sigma=\frac{1}{2 \pi} \int_{\Gamma} \mu(\sigma) \frac{1}{z-t(\sigma)} d \sigma \\
=\frac{1}{2 \pi} \int_{\Gamma} \mu(\sigma) e^{-i \alpha(\sigma)} \frac{1}{z-t(\sigma)} d t=-\frac{1}{2 \pi} \int_{\Gamma} \hat{\mu}(t) \frac{1}{t-z} d t
\end{gathered}
$$

where $\hat{\mu}(t)=\mu(\sigma) e^{-i \alpha(\sigma)}$. In $P_{E}[\mu](x)$ we may take either $\mu \equiv \mu_{1}$ or $\mu \equiv \mu_{2}$. Therefore, to study functions $P_{c}\left[\mu_{1}\right](x), P_{s}\left[\mu_{2}\right](x)$, it is sufficient to study a function $P_{E}[\mu](x)$ and to take its real or imaginary part.

Theorem 5. Let $\Gamma$ be an open arc of class $\mathbb{C}^{1, \lambda}, \lambda \in(0,1], t=t(\sigma)=\left(y_{1}(\sigma)+i y_{2}(\sigma)\right) \in$ $\Gamma$. Let $\mu(\sigma) \in \mathbb{C}_{q}^{\omega}[a, b], \omega \in(0,1], q \in[0,1)$. Then

1) the function $P_{E}[\mu](x)$ belongs to $\mathbb{C}^{0}\left(\overline{\mathbb{R}^{2} \backslash \Gamma} \backslash X_{\Gamma}\right) \cap \mathbb{C}^{2}\left(\mathbb{R}^{2} \backslash \Gamma\right)$, where $X_{\Gamma}=x(a) \cup x(b)$ is a set of endpoints of $\Gamma$; 
2) the limiting values of the function $P_{E}[\mu](x)$ on $\Gamma^{ \pm}$in the interior points are given by the formula

$$
\begin{gathered}
\left.P_{E}[\mu](x)\right|_{x(s) \in \Gamma^{ \pm}}=-i\left( \pm \frac{1}{2} e^{-i \alpha(s)} \mu(s)+\frac{1}{2 \pi i} \int_{\Gamma} \frac{\hat{\mu}(t)}{t-t_{0}} d t\right) \\
=-i\left( \pm \frac{1}{2} e^{-i \alpha(s)} \mu(s)-\frac{1}{2 \pi i} \int_{\Gamma} \frac{\mu(s) e^{-i \psi(x(s), y(\sigma))}}{|x(s)-y(\sigma)|} d \sigma\right)
\end{gathered}
$$

where $t_{0}=t_{0}(s)=\left(x_{1}(s)+i x_{2}(s)\right) \in \Gamma$ and the integral is understood in the sense of a principal value;

3 ) for any point $x \notin \Gamma$ placed in a small neighbourhood of the endpoint $x(d)$ ( $d=a$ or $d=b$ ), the following estimate holds:

$$
\left|P_{E}[\mu](x)\right| \leq \frac{\text { const }}{|x-x(d)|^{\delta}},
$$

where $\delta=q$ if $q \in(0,1)$ and $\delta$ is an arbitrary number from the interval $(0,1)$ if $q=0$.

REmark. The definition of the functional class $\mathbb{C}^{0}\left(\overline{\mathbb{R}^{2} \backslash \Gamma} \backslash X_{\Gamma}\right)$ is given in the remark to Corollary 1.

Proof of the theorem. According to Lemma 2(2), if $\mu(\sigma) \in \mathbb{C}_{q}^{\omega}[a, b], \omega \in(0,1], q \in$ $[0,1)$, and if $\hat{\mu}(t)=\mu(\sigma) e^{-i \alpha(\sigma)}$. Then $\hat{\mu}(t) \in \mathbb{C}_{q}^{\beta}(\Gamma), \beta=\min \{\omega, \lambda\}$. Using properties of Cauchy-type integrals from [1, sections $15,16,22]$, we observe that points 1) - 3) of the theorem hold for the Cauchy-type integral $P_{E}[\mu](x)$ with the density $\hat{\mu}(t)$.

Theorem 6. Let $\Gamma$ be an open arc of class $\mathbb{C}^{1, \lambda}, \lambda \in(0,1]$;

$$
\mu(\sigma)=\frac{\eta(\sigma)}{|\sigma-d|^{q}}, \quad q \in(0,1) ; \quad \eta(\sigma) \in \mathbb{C}^{0, \omega}[a, b], \quad \omega \in(0,1] ;
$$

$d=a$ or $d=b$. Let

$$
\hat{\nu}(t)=\hat{\nu}(t(\sigma))=\left(\frac{t(\sigma)-t(d)}{|\sigma-d|}\right)^{q} \eta(\sigma) e^{-i \alpha(\sigma)} .
$$

Then $\hat{\nu}(t) \in \mathbb{C}^{0, \beta}(\Gamma)$, where $\beta=\min \{\omega, \lambda\}$ and for points $z=x_{1}+i x_{2}$, lying near $D=t(d)$ outside $\Gamma$, the following asymptotic formula holds:

$$
P_{E}[\mu](x)=\mp \frac{e^{ \pm q \pi i}}{2 \sin (\pi q)} \frac{\hat{\nu}(D)}{(z-D)^{q}}+\Theta_{0}(z),
$$

the upper sign is taken if $d=a$, while the lower sign if $d=b$. The function $\Theta_{0}(z)$ is continuous as $z \rightarrow D$ if $\beta>q$. If $0<\beta \leq q<1$, then for $z$, lying near $D=t(d)$ outside $\Gamma$, the following estimate holds:

$$
\left|\Theta_{0}(z)\right| \leq \frac{\text { const }}{|z-D|^{q_{1}}},
$$

where $q_{1}$ is an arbitrary number such that $q_{1} \in(q-\beta, q)$. Moreover, $\Theta_{0}(z)$ is analytic in the neighbourhood of $D$ cut along $\Gamma$. By $(z-D)^{q}$ we denote the branch that is analytic in the neighbourhood of $D$ cut along $\Gamma$ and that takes the value $(t-D)^{q}$ on $\Gamma^{+}$. In addition,

$$
\hat{\nu}(D)=\lim _{\sigma \rightarrow d} \hat{\nu}(t(\sigma)), \quad \text { where } \quad \sigma \in(a, b) .
$$


The proof of the theorem follows from the properties of Cauchy-type integrals presented in [1, sections $22,6.3,15.2]$.

\section{REFERENCES}

[1] Muskhelishvili, N.I. Singular integral equations. Nauka, Moscow, 1968. (In Russian; English translation: Noordhoff, Groningen, 1972.) MR0355494 (50:7968)

[2] Popov, A.N. Application of potential theory to solving the linearized Navier-Stokes system of equations in a two-dimensional case. Trudy MIAN, 1971, t.116, pp.162-180. (In Russian). MR0364909 $(51: 1163)$

[3] Power, H. The completed double layer boundary integral equation method for two-dimensional Stokes flow. IMA Journal of Applied Mathematics, 1993, v.51, pp.123-145. MR.1244192 (94i:76020)

[4] Krutitskii, P.A. The Dirichlet problem for the Helmholtz equation in the exterior of cuts in the plane. Comput. Math. Math. Phys., 1994, v.34, No.8/9, pp.1073-1090. MR.1300397 (95f:35046)

[5] Vladimirov, V.S. Equations of Mathematical Physics. Nauka, Moscow, 1981. (In Russian; English translation of 1st edition: Marcel Dekker, N.Y., 1971.)

[6] Gakhov, F.D. Boundary value problems. Fizmatlit, Moscow, 1963. (In Russian; English translation: Pergamon Press, Oxford; Addison-Wesley, Reading, Mass., 1966.) MR0198152 (33:6311)

[7] Shilov, G.E. Mathematical Analysis. Special course. Fizmatlit, Moscow, 1960. (In Russian.)

[8] Pozrikidis, C. Boundary integral and singularity method for linearized viscous flow. Cambridge University Press, Cambridge, 1992. MR.1156495 (93a:76027)

[9] Varnhorn, W. The Stokes equations. Akademie Verlag, Berlin, 1994. MR1282728 (95e:35162) 